\title{
Shōnishin bei Kindern
}

\section{und Jugendlichen}

\author{
Die Behandlung erfolgt auf der Ebene des energetischen Entwicklungsalters - Besonders wirksam \\ ist sie bei Regulationsstörungen und funktionellen Störungen
}

Thomas Wernicke

\section{Shōnishin - von der Tradition in die Moderne}

In Japan etablierte sich eine eigenständige Medizintradition, die sich den dortigen gesellschaftlichen und wirtschaftlichen Gegebenheiten bis zum heutigen Tag angepasst hat und als „Japanisch“ bezeichnet werden kann. Ihren Anfang nahm diese Entwicklung ab dem 16. Jahrhundert, als es zu einer zunehmend kritischen Betrachtungsweise der chinesischen Medizin kam. Diese Entwicklung verstärkte sich durch die Ankunft der ersten Europäer und die damit aufkommende Auseinandersetzung mit der westlichen Chirurgie und Anatomie. Auch setzte die Verbreitung der westlichen Medizin auf der Basis von Naturwissenschaften ab Mitte des
19. Jahrhunderts neue Impulse. In diese Tradition ist auch die Kinderakupunktur Shōnishin einzureihen.

Wörtlich übersetzt bedeutet der Begriff Shōnishin „Kindnadelung“ (shōni = Kind; shin = Nadel/Nadelung). Und mit Akupunkturnadeln wurden Kinder in Japan auch behandelt, indem an sogenannten azeketsu (Ach-das-ist-es!)-Punkten und tsubos (Akupunkturpunkte) gestochen wurde. Aus der bei Kindern zunächst traditionell mit $\mathrm{Na}$ deln durchgeführten Akupunktur entwickelte sich zu Beginn des 20. Jh. eine nadellose und damit nicht-invasive Behandlungsmethode [1].

Eine Weiterentwicklung erfuhr Shōnishin im Westen. Durch Einbindung wissenschaft- licher Erkenntnisse über kindliche Entwicklung und der energetischen Entwicklung in das diagnostische Konzept eröffneten sich neue Behandlungsstrategien - und damit erweitererte sich das Behandlungsspektrum von Babys und Kindern nun auch auf Jugendliche und Erwachsene [2]. Durch diese dem heutigen Wissensstand angepasste Form wurden neue Standards in der Behandlung mit Shōnishin geschaffen. Nicht zuletzt auch deswegen können wir außerhalb Japans, besonders im europäischen, speziell im deutschsprachigen (Deutschland, Österreich, Schweiz) und englischsprachigen Raum(USA, Kanada, Großbritannien und Australien), seit den letzten zwei Jahrzehnten ein stetig wachsendes Interesse an Shōnishin beobachten. 


\section{Zusammenfassung}

Seit der Einführung der Akupunktur aus China nach Japan werden Kinder auch in Japan mit Akupunktur behandelt - daher der japanische Begriff Shōnishin (shöni = Kind; shin = Nadel/Nadelung). Zu Beginn des 20. Jh. veränderte sich die Behandlungstechnik: Die einst invasive, stechende Akupunktur entwickelte sich zu einer sanften, nicht-invasiven Behandlungsmethode.

Keine 100 Jahre später erfuhr Shōnishin eine nochmalige Weiterentwicklung und eröffnete durch die Verbindung der sino-japanischen Medizintradition mit dem heutigen Wissensstand der Entwicklungsphysiologie neue Behandlungsstrategien.

Kurzgefasst kann diese Weiterentwicklung folgendermaßen zusammengefasst werden: Entsprechend den motorischen, sensorischen, emotionalen und sozialen Entwicklungsschritten des Kindes baut auch jeder energetische Entwicklungsschritt auf einem jeweils vorher erworbenen Entwicklungsschritt auf. Dabei spielen insbesondere bestimmte Gruppierungen von Meridianen eine übergeordnete Rolle. Sie stellen ein Kommunikationsnetzwerk dar, welches den Einzelnen mit seiner Außenwelt verbindet. Über diese Verbindung ermöglichen die Meridiane die Integration von Reflexen und Reizen. Damit sind sie auch für die Entwicklung von Haltung, Bewegung, Persönlichkeits- und Verhaltensmuster zuständig. Demnach sind mehrere Entwicklungsschritte zur Entfaltung der Meridiane notwendig, bevor diese als die bekannten Einzelmeridiane mit ihren Funktionskreisen in Erscheinung treten.

Dieses Wissen über die Vernetzung zwischen Meridiansystem und Neurophysiologie, Entwicklungspsychologie und -physiologie eröffnet neue Blickwinkel auf die kindliche Entwicklung. Spannend wird es, wenn erkennbar wird, welche Meridiangruppierung welchen Entwicklungsschritt steuert und was passiert, wenn auf dieser Vernetzungsebene eine Störung auftritt. Dieses Wissen bildet die Grundlage für die Behandlung von Kindern jeglicher Altersstufe bis zum Erwachsenen mit Shōnishin.

\section{Energetische Entwicklung auf einen Blick}

Wir gehen davon aus, dass alle 12 Hauptmeridiane beim Erwachsenen sich vollständig entwickelt haben. Doch bevor es so weit ist, müssen diese einen Entwicklungsprozess durchlaufen, der sich in drei fließend ineinander übergehenden Phasen vollzieht [2].

1. Die erste Phase beginnt mit der Geburt und wird durch die Entwicklungsebene der Drei Familien geprägt. Hier wirken jeweils vier noch nicht entfaltete Meridiane als Gruppe in ihrer Gesamtheit - als Einzelmeridian sind sie noch unreif.

2. Dieser Phase schließt sich ab dem freien Laufen die zweite Phase an - die Entwicklungsebene der Sechs Keiraku (jap. keiraku = Leitbahn). Diese wird initiiert durch die Aufrichtung vom Vierfüßlerstand in den Zweifüßlerstand. Dadurch kommt es zu einer Oben-Unten-Verbindung der Arm- und Beinmeridiane innerhalb einer jeden Meridiangruppe.

3. Mit Erlangen der Schulreife tritt das Kind in die dritte Phase ein und befindet sich nun auf der Ebene der Fünf Wandlungsphasen. Erst mit dem Erwachsenwerden haben sich die zwölf Hauptmeridiane vollständig entfaltet. rung seiner Motorik zu arbeiten. Es entdeckt seinen sportlichen Ehrgeiz - nicht mehr das Spielerische, sondern zunehmend die Ansprüche an die eigenen Leistungen stehen im Vordergrund. Wendigkeit, Beweglichkeit, Kraft, Schnelligkeit oder auch körperliche Grenzerfahrungen sind die körperlichen Herausforderungen, die das Kind jetzt gezielt sucht. Gleichzeitig findet eine Identifizierung mit dem eigenen Geschlecht mit zunehmend typischem Verhalten statt. Lehrer und Mitschüler werden zu neuen Bezugspersonen.

Auch auf emotionaler Ebene findet ein „Feintuning“ statt. Die plötzlichen und ungefilterten emotionalen Ausdrucksmöglichkeiten im Kindergarten- und Vorschulalter entwickeln sich zu situationsangepassten fein abgestuften Äußerungen. Damit ist das Kind in der Lage, seine Innenwelt der Außenwelt adäquat anzupassen und die Außenwelt differenzierter wahrzunehmen mit anderen Worten: Es verbessert seine soziale Kompetenz.

Auf energetischer Ebene standen bisher die Keiraku im Vordergrund. Die Oben-Un-

\section{Kinder durchlaufen drei Entwicklungsphasen, bis}

\section{sich mit dem Erwachsenwerden alle zwölf Haupt- meridiane vollständig entfaltet haben. Chronologi- sches Alter und energetisches Entwicklungsalter}

\section{stimmen dabei nicht immer überein!}

Damit sind die energetischen Entwicklungsebenen bei regelrechter Entwicklung in folgenden Entwicklungsphasen zu erwarten:

- Babys und Kleinkinder bis zum freien Laufen - Entwicklungsebene Drei Familien

- Klein- und Vorschulkinder - Entwicklungsebene Sechs Keiraku

- Schulkinder bis zum Abschluss der Pubertät - Entwicklungsebene Fünf Wandlungsphasen

Die Bedeutung der energetischen Entwicklung im therapeutischen Ansatz

Mit der Schulreife beginnt das Kind verstärkt an der Verfeinerung und Spezialisie-
ten-Verbindungen der jeweiligen Yin- und Yang-Meridiane stellten ab dem Zeitpunkt des freien Laufens die Grundlage dar, um die neue motorische Erfahrung der Aufrichtung energetisch zu ermöglichen. Ab Schulalter sollten alle sechs Keiraku in ihrem Zusammenspiel automatisierte und ökonomische Bewegungsabläufe für den Alltag zur Verfügung stellen können. Das Kind ist nun in der Lage, auf einem Bein zu stehen und zu hüpfen, es kann auf einen Baum klettern, es kann rückwärtslaufen, es kann balancieren und es kann Fahrrad fahren - um nur einige Bewegungsbeispiele zu nennen.

Um auf Meridianebene seine Innenwelt der Außenwelt anzupassen, orientieren sich zunehmend die „inneren“ Yin-Meridiane nach den „äußeren“ Yang-Meridianen und 
umgekehrt die Yang-Meridiane nach den Yin-Meridianen. So können sich durch die Yang-Meridiane äußere Einflüsse auf das Innenleben des Kindes auswirken, und über die Yin-Meridiane kann die innere Befindlichkeit als emotionaler Ausdruck in die Außenwelt transportiert werden. Damit findet ein ständiger Abgleich zwischen äußeren Anforderungen und dem inneren Bedürfnis statt was nicht immer konfliktlos geschieht.

Diese Erfahrung ermöglicht es dem Kind, sein zukünftiges Verhalten an die jeweilige Situation zu modifizieren und anzupassen oder auch nicht. Jetzt hat das Schulkind, je nach Situation, Zugriff auf die passende energetische Entwicklungsebene (Familie, Keiraku, Wandlungsphasen). Dieser Zugriff ist ihm aber verwehrt, wenn die energetische Verbindung auf der entsprechenden Entwicklungsebene nicht zur Ausreifung gelangt ist.

Basis des therapeutischen Ansatzes bildet die Grundannahme, dass jeder Mensch seine Potenziale mit auf die Lebensreise bekommt, damit die drei energetischen Entwicklungsebenen Familie, Keiraku, Wandlungsphasen im entsprechenden Alter ausreifen können [3]. Doch energetisches und tatsächliches Alter stimmen oft nicht überein.

\section{Beispiel}

Ein siebenjähriges Schulkind sollte vom Alter her die Entwicklungsebene Wandlungsphasen erreicht und damit entsprechende altersgemäße Handlungsstrategien zur Verfügung haben. Doch ein Blick ins Klassenzimmer zeigt ein anderes Bild. Hier beobachten wir auffallend viele Kinder, die durch Unruhe, mangelnde Aufrichtung, aufbrausendes, zurückgezogenes oder ängstliches Verhalten in Erscheinung treten. Ihre Verhaltensmuster zeigen an, dass sie energetisch noch auf der Keiraku-Entwicklungsebene verharren.

Dies zu erkennen ist wichtig für die diagnostische Einschätzung! Das Verhalten von Schulkindern verleitet nämlich zu oft dazu, dass Diagnosen schnell und voreilig gestellt werden - zum Beispiel die Diagnose „Verhaltensauffälligkeit“ oder „ADHS“. Dadurch werden Kinder leichtfertig für ihren weiteren Schul- und Lebensweg abgestempelt. Doch diesem auffälligen Verhalten kann zugrunde liegen, dass diesen Kindern keine anderen Handlungsmuster zur Verfügung stehen - eben weil sie sich energetisch nicht weiterentwickelt haben. Gründe dafür gibt es viele. Hier die am häufigsten vorkommenden: zu wenig Bewegung, zu viel Medienkonsum, früh einsetzender Leistungsdruck, hohe Erwartungshaltung, Tod von Oma oder Opa, Umzug der Familie, Trennung der Eltern oder Platz machen für ein neues Geschwisterkind.

\section{Behandlung}

Deshalb richtet sich die ShōnishinBehandlung nicht nach dem chronologischen Alter des Kindes, sondern danach, auf welcher energetischen Entwicklungsebene eine vorliegende Symptomatik erstmals aufgetreten ist und das Kind sich deshalb energetisch nicht weiterentwickeln konnte. Diese Entwicklungsebene bedarf der Unterstützung bzw. einer Nachreifung, da sie beim ersten Auftreten der Symptomatik offensichtlich geschwächt war oder sich noch nicht altersgemäß entwickelt hatte, andernfalls hätte z.B. durch Selbstregulation das Entstehen der Symptomatik verhindert werden können. Darum werden die Meridiane in ihrer entsprechenden Meridian-Konstellation, wie sie für die infrage kommende Entwicklungsebene typisch ist, stimuliert.

\section{Beispiel 1}

Ist die behandlungsbedürftige Symptomatik bei einem Jugendlichen, z. B. eine erhöhte Infektanfälligkeit, im Kleinkind-/Kindergartenalter erstmalig aufgetreten, dann betrifft dies die Keiraku-Entwicklungsebene, obwohl der Jugendliche vom Alter her sich im Wandlungsphasen-Alter befindet. Deshalb werden in diesem Fall die Meridiane in ihrer Keiraku-Konstellation stimuliert, d.h. im Verlauf des jeweiligen Yin-Meridianpaars vom Bein zum Arm (hier: Milz $\rightarrow$ Lunge) auf beiden Körperseiten sowie des Yang-Meridianpaars vom Arm zum Bein (hier: Dickdarm $\rightarrow$ Magen bds.).

\section{Beispiel 2}

Traten bei einer Zwölfjährigen Schlafstörungen durch Gedankenandrang erstmalig im Schulalter auf, dann werden die Meridiane im Verlauf der entsprechenden Wandlungsphasen behandelt, d.h. das jeweilige Yin-/ Yang-Meridianpaar an beiden Armen (hier: Herz $\rightarrow$ Dünndarm = Feuer) und das Yang- $/$ Yin-Meridianpaar (hier: Blase $\rightarrow$ Niere $=$ Wasser) an beiden Beinen.

In beiden Beispielen wird die Streichtechnik angewendet; diese folgt, wie gene- rell bei jeder Meridianbehandlung, dem Ki(Qi-)Fluss des jeweiligen Meridians (Abb. 1).

Wie bereits angedeutet wird im Shōnishin nicht mit Nadeln gestochen. Vielmehr wird anstelle von Nadeln mit verschiedenartigen Spezialinstrumenten (Abb. 2), ohne zu stechen, auf der Körperoberfläche gearbeitet. Die Behandlung findet also direkt auf der Haut statt, ohne diese zu verletzen.

Neben den bereits erwähnten Streichungen im Bereich bestimmter Meridiane oder auch Körperareale kommen weitere Behandlungstechniken wie Druck- und Klopftechniken (Abb. 3) an bestimmten Reflexzonen und Meridianabschnitten oder Vibrationstechniken an ausgewählten Akupunkturpunkten (Abb. 4) zur Anwendung. Mit diesen shōnishinspezifischen Behandlungstechniken können oberflächliche wie

\section{Fallbeispiel}

Sophie (Name redaktionell geändert) kannte ich noch aus der Zeit, als sie ein Baby war. Damals, vier Monate alt, behandelte ich sie wegen eines KiSS-Syndroms, wobei alle Auffälligkeiten bereits nach der zweiten Behandlung nicht mehr in Erscheinung traten.

Nun wurde sie mir im Alter von siebeneinhalb Jahren erneut vorgestellt. Sie ging in die erste Klasse, und auf Anraten der Lehrerin durchlief sie seit sechs Monaten eine Therapie wegen „emotionaler Entwicklungsstörung“. Ihre Symptome äußerten sich in zunehmender Unausgeglichenheit, Neigung zur Überreaktion, Konzentrationsproblemen und einem Gefühl, nicht geliebt zu werden. Begonnen hatte alles vor vier Jahren mit der Geburt ihrer Schwester.

Ihre Symptomatik zeigte mir an, dass sie energetisch noch nicht die Wandlungsphasenebene erreicht hatte - vielmehr schien sie noch im Keiraku-Verhalten zu verharren, ausgelöst durch die Geburt ihrer Schwester. Offenbar führte dieses Ereignis zu einer Störung ihrer KeirakuEntwicklung.

Deshalb behandelte ich dreimal ihre Keiraku ( $\mathrm{Di}, \mathrm{Ma}, \mathrm{Mi}$, Lu). Eine Woche nach der letzten Behandlung beschrieb ihr Vater mir die Veränderungen folgendermaßen: Bereits seit der zweiten Behandlung zeige sie zunehmend bessere Selbstkontrolle, sie sei deutlich ausgeglichener. würde mehr lachen und suche die Nähe zum Kuscheln. 


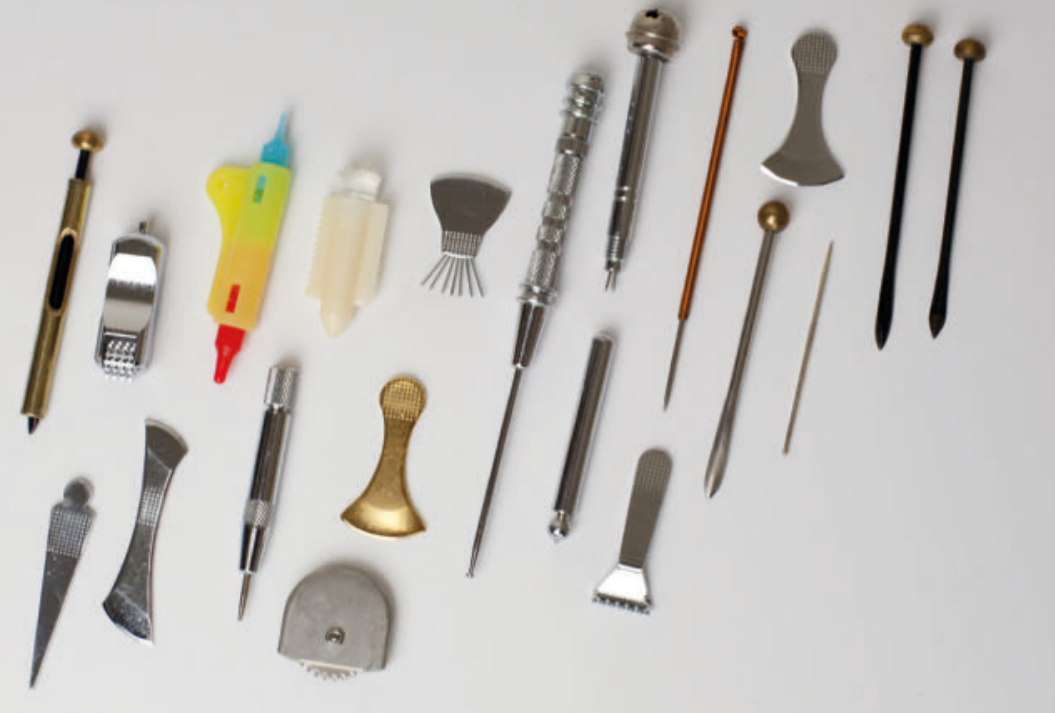

Abb. 2 Eine kleine Auswahl an Shōnishin-Instrumenten. Quelle: Thomas Wernicke

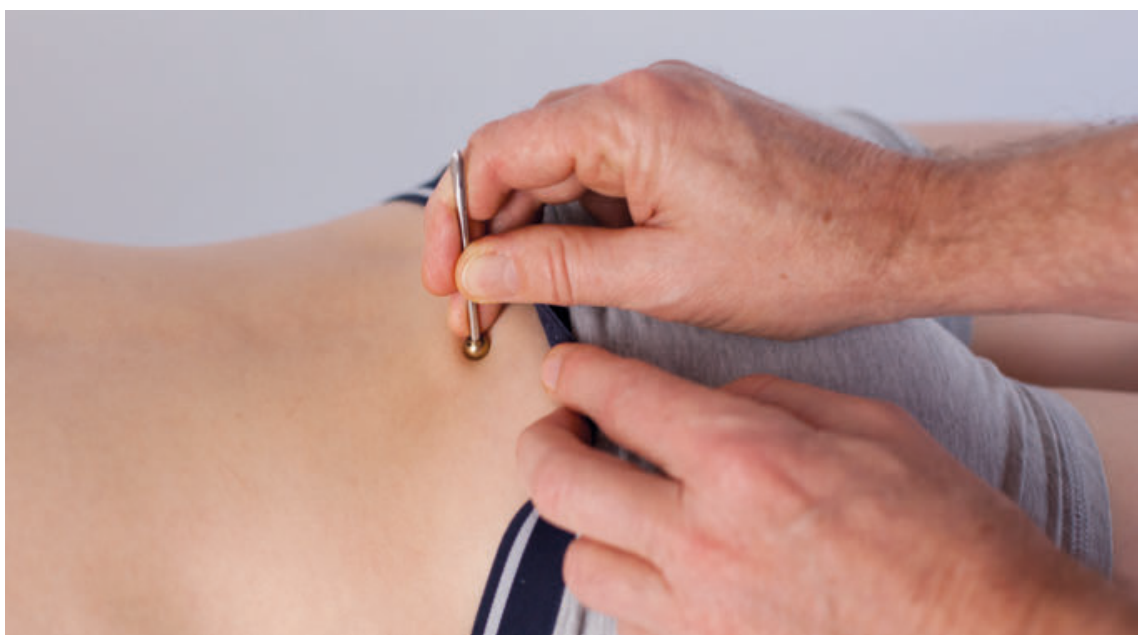

Abb. 3 Klopftechnik auf dem Sakrum, um über Stimulation der Tiefenwahrnehmung (Propriozeption) die Blasenkontrolle zu verbessern (gleicher Patient). Quelle: Thomas Wernicke

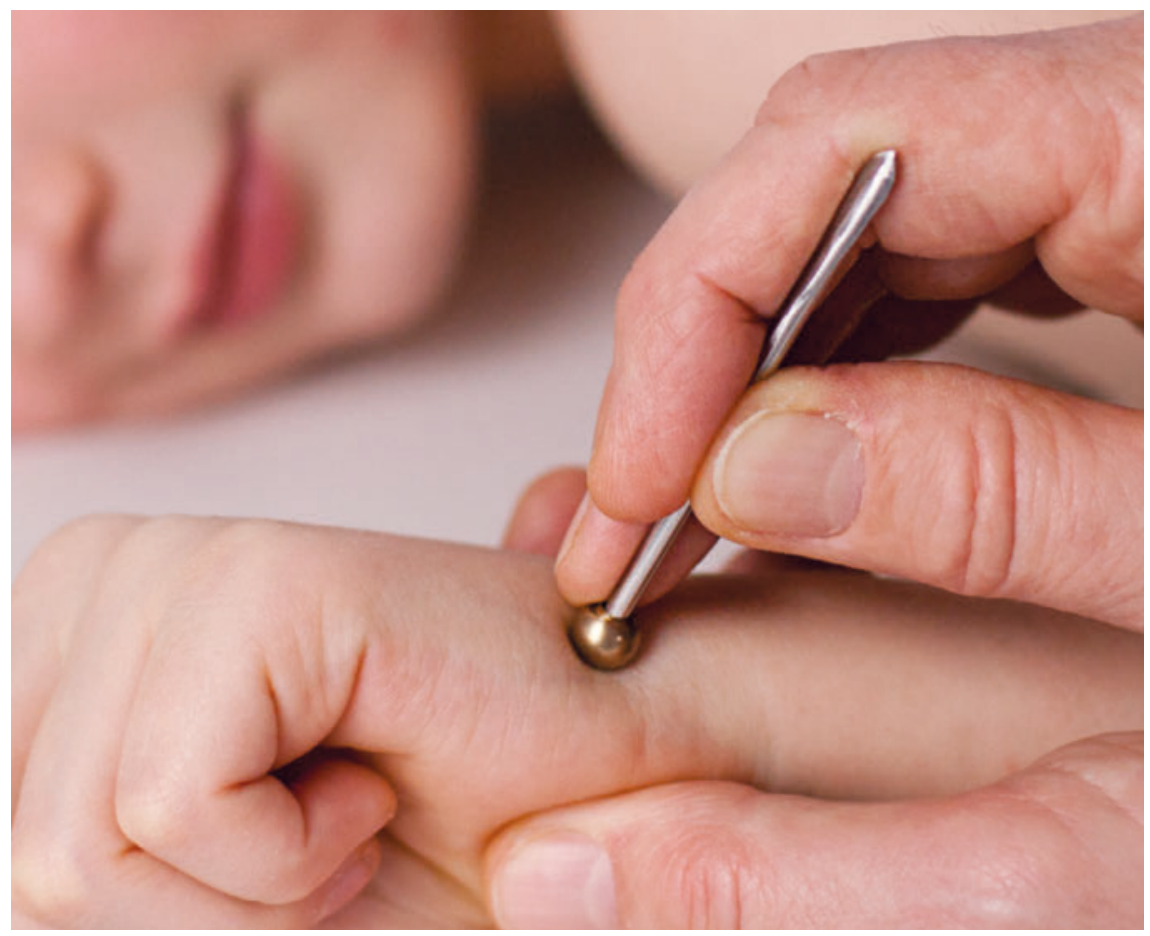

Abb. 4 Vibrationstechnik des Akupunkturpunktes Dü 4 im Rahmen der Behandlung einer ADHS-ähnlichen Symptomatik mit Rastlosigkeit. Quelle: Thomas Wernicke

\section{Shōnishin Praxis zkm}

auch tiefere Schichten in und unter der Haut erreicht werden, ohne diese zu penetrieren [4].

Abhängig davon, auf welcher Gewebetiefe bzw. mit welcher Behandlungstechnik die Behandlung durchgeführt wird, kommt es zu unterschiedlichen Behandlungseffekten. Denn gewisse Reizqualitäten werden nur in bestimmten Hautschichten wahrgenommen und zu unterschiedlichen Rückenmarkssegmenten weitergeleitet, was wiederum zu unterschiedlichen Reizantworten führt [5]. Damit wird ein Arbeiten sowohl auf neuroanatomischer Ebene als auch auf energetischer Ebene ermöglicht.

Die reine Behandlungszeit beträgt bei Säuglingen weniger als 5 Minuten und steigert sich mit zunehmendem Alter auf 8-10 Minuten bei großen Kindern und Erwachsenen. Bei funktionellen und akuten Beschwerden wird Shōnishin in der Regel 1-mal pro Woche, in seltenen Fällen auch täglich durchgeführt, bei chronischen alle 1-2 Wochen.

\section{Wirkung von Shōnishin}

Die Stärke einer Shōnishin-Behandlung besteht darin, die „Mitte“ wiederherzustellen, z.B. durch Regulation des vegetativen Nervensystems (VNS). Mittels Herzfrequenzvariabilitätsmessungen (HRV) lässt sich das eindrucksvoll nachweisen [3]. In den folgenden Beispielen zeigen die drei linken Balken im jeweiligen Diagramm die HRV-Analyse unmittelbar vor Behandlungsbeginn (violett = Herzfrequenz [HF], rot = Sympathikusaktivität [SI], blau=Parasympathikusaktivität [RMSSD]). Die drei rechten Balken sind die Aufzeichnungen unmittelbar nach der Behandlung.

\section{Fall 1}

Leon (Name redaktionell geändert), Junge, 9 Jahre (Abb. 5).

Diagnose/Symptomatik: seit Kleinkindalter regelmäßige Kopf- und Bauchschmerzen, Schlafstörung, nächtliche Angstzustände, setzt sich zunehmend unter Leistungsdruck

Die HRV-Analyse vor Behandlung zeigt ein deutliches Übergewicht des Sympathikus gegenüber dem Parasympathikus. Daraufhin wurde die Meridian-Behandlung der vorderen Keiraku (Mi-Lu und Di-Ma) durchgeführt (Dauer: $3 \mathrm{~min}$ ), anschließend eine zweite HRV-Analyse. 


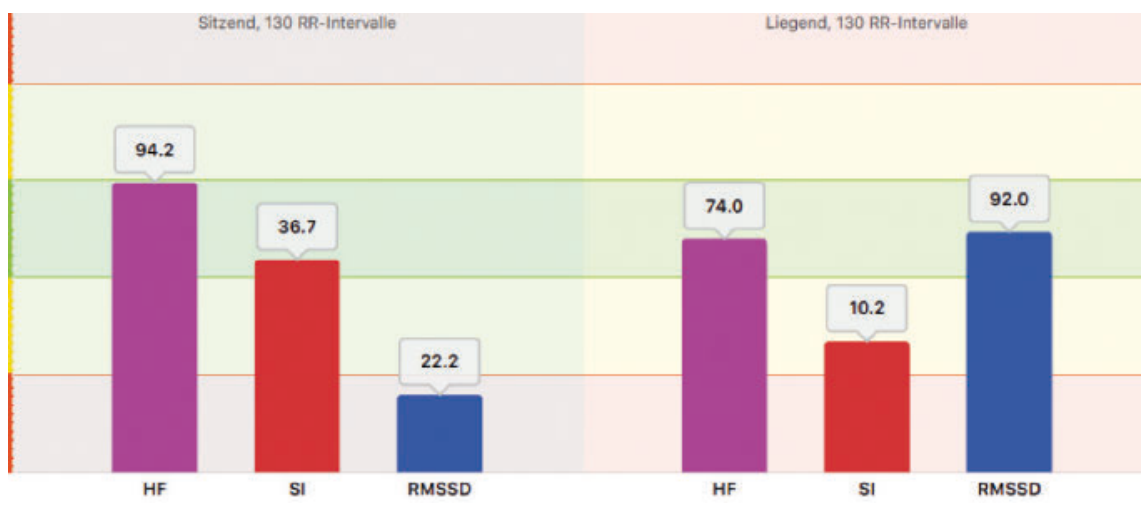

Abb. 5 HRV-Analyse vor und nach Shōnishin-Behandlung: $a$. vor Behandlung, b. nach Behandlung (violett = Herzfrequenz [HF], rot = Sympathikusaktivität [SI], blau = Parasympathikusaktivität [RMSSD]). Quelle: Thomas Wernicke

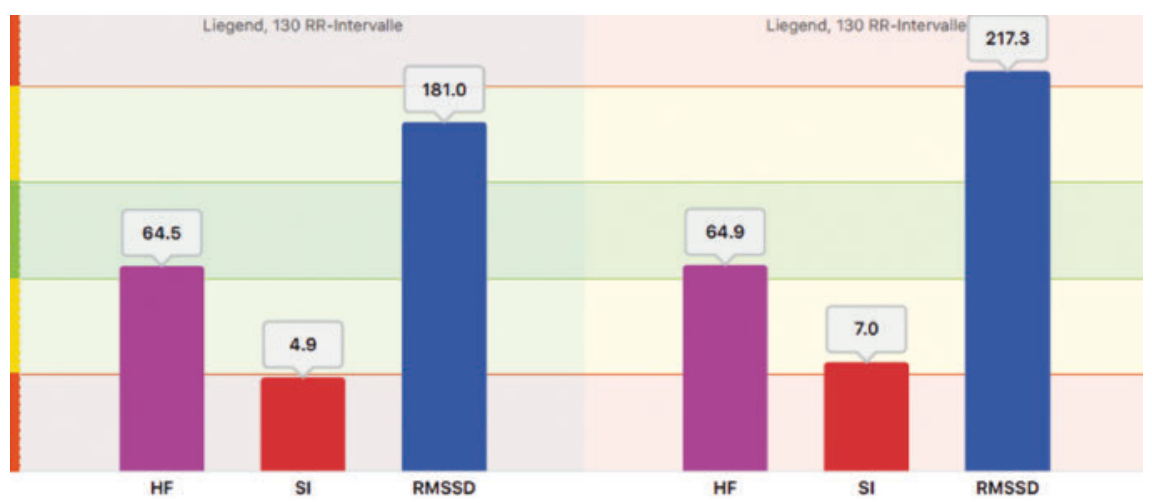

Abb. 6 HRV-Analyse vor und nach Shōnishin-Behandlung: $a$. vor Behandlung, b. nach Behandlung (violett = Herzfrequenz [HF], rot = Sympathikusaktivität [SI], blau = Parasympathikusaktivität [RMSSD]). Quelle: Thomas Wernicke

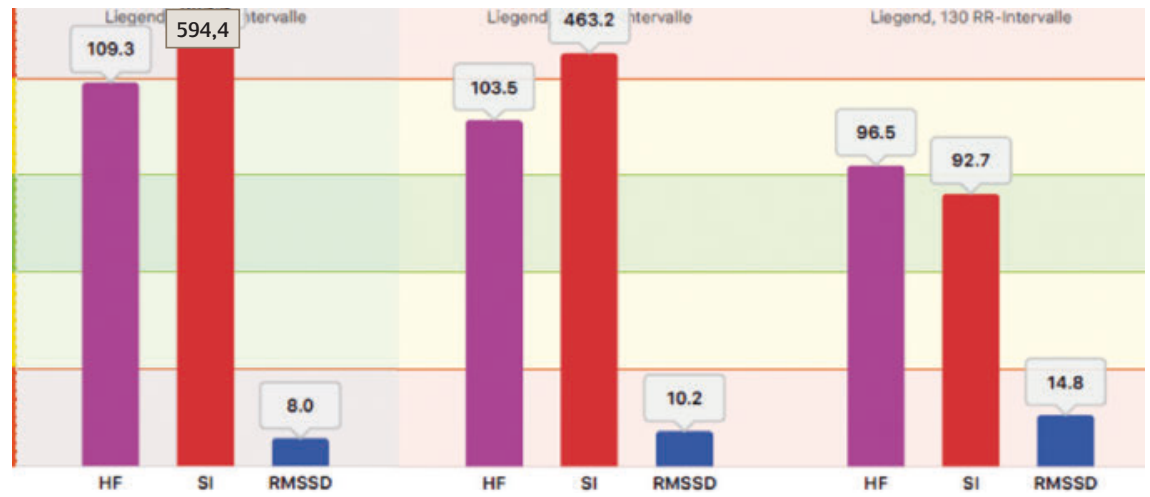

Abb. 7 HRV-Analyse vor und nach Shōnishin-Behandlung: $a$. vor Behandlung, b. nach Behandlung (violett = Herzfrequenz [HF], rot = Sympathikusaktivität [SI], blau = Parasympathikusaktivität [RMSSD]). Quelle: Thomas Wernicke

Ergebnis: Unmittelbare Reaktion des VNS durch Senkung der Sympathikusaktivität und Steigerung der Parasympathikusaktivität sowie Absenkung der Herzfrequenz.

Leon wurde insgesamt 5-mal im wöchentlichen Rhythmus behandelt. Nach der 2. Behandlung traten keine nächtlichen Angstzustände mehr auf, entsprechend war auch sein Schlaf ungestört. Ab der 5. Behandlung verschwanden die Kopf- und Bauchschmerzen und traten bisher nicht mehr auf.

\section{Fall 2}

Mia (Name redaktionell geändert), Mädchen, 12 Jahre (Abb. 6)
Diagnose/Symptomatik: primäre Enuresis, „komatöser“ Schlaf

Durch die Shōnishin-Behandlung wurde ein bereits erhöhter Parasympathikus stimuliert, was zu einem weiteren Ungleichgewicht bei unveränderter Herzfrequenz führte - dies zeigte sich auch an der Symptomatik: Es trat keine Besserung des Einnässens ein.

\section{Fall 3}

Emma (Name redaktionell geändert), Mädchen, 11 Jahre (Abb. 7)

Diagnose/Symptomatik: Schlafstörung, Verlustängste

Schon während der Behandlung (mittlere drei Balken) milderte sich die deutlich erhöhte Sympathikusaktivität leicht ab. Nach der Behandlung bestand zwar immer noch ein ausgeprägtes Ungleichgewicht zwischen Sympathikus und Parasympathikus, aber beide Teile des VNS näherten sich an. Die Herzfrequenz senkte sich deutlich ab.

Ergebnis: Verlustängste und die dadurch hervorgerufenen Schlafstörungen traten ab der zweiten Behandlung nur noch sporadisch auf.

\section{Fazit}

Die kindlichen Entwicklungsschritte im Spiegel einer Meridianreifung zu sehen führt zu einer erweiterten Herangehensweise in der Behandlung von Kindern und Jugendlichen. Störungen oder Auffälligkeiten lassen sich durch diese Sichtweise anders einordnen, wenn chronologisches Alter und energetisches Entwicklungsalter berücksichtigt werden. Behandelt werden demnach Kinder und Jugendliche nicht unbedingt ihrem eigentlichen Alter entsprechend, sondern auf der Ebene ihres energetischen Entwicklungsalters, auf der erstmalig das behandlungsbedürftige Problem auftrat. Chronologisches Alter und energetisches Entwicklungsalter stimmen nicht immer überein!

Mit Shōnishin lassen sich besonders Regulationsstörungen und funktionelle Störungen wirksam behandeln. Dies gilt insbesondere dann, wenn Wachstum, Hormone oder äußere Einflüsse eine Regulation stören oder gar verhindern.

Interessenkonflikt: Der Autor erklärt, dass keine Interessenkonflikte bestehen.

http://dx.doi.org/10.1055/a-1201-8779 


\section{Literatur}

$\overline{1}$ Michel W. Historical background: Treating children in Japan. In: Wernicke T. Shōnishin - The Art of non-invasive Paediatric Acupuncture. London: Singing Dragon; 2014

$\overline{2}$ Wernicke T, Kalbantner-Wernicke K. Kinderakupunktur mit der entwicklungsphysiologisch orientierten Shōnishin-Methode. Dt Ztschr $\mathrm{f}$ Akup 2009; 49 (2): 18-24

$\overline{3}$ Wernicke T. Shōnishin - Entwicklungsphysiologie und Meridianentfaltung in der Kinderakupunktur. München: Kiener; 2020

$\overline{4}$ Wernicke T. Shōnishin - The art of non-invasive paediatric acupuncture. London: Singing Dragon; 2014

5 Wernicke T. Shōnishin - Nicht-invasive Akupunktur im Lichte der Segment-Akupunktur. Dt Ztschr f Akup 2019; 62 (1): 9-11

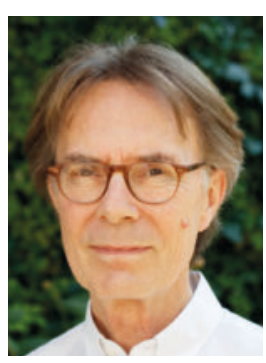

\section{Thomas Wernicke}

Facharzt für Allgemeinmedizin

Vorsitzender der Internationalen Gesellschaft für Traditionelle Japanische Medizin (IGTJM)

Alte Dorfgasse 13

65239 Hochheim

tw@therapeuticum.de

Thomas Wernicke ist Facharzt für Allgemeinmedizin und ärztlicher Leiter des Therapiezentrums therapeuticum rhein-main. Als international anerkannter und gefragter Experte für Shōnishin (nadellose japanische Kinderakupunktur) ist er in vielen Ländern Europas, Asiens (inkl. Japan), in Australien und in den USA als Ausbildungsleiter tätig. Neben zahlreichen wissenschaftlichen Veröffentlichungen hat er mehrere Fachbücher zum Thema geschrieben. Er ist Mitglied der Japanischen Wissenschaftlichen Gesellschaft für Kinderakupunktur (Nihon Shoni Hari Gakkei) und Gründungsmitglied der Internationalen Gesellschaft für Traditionelle Japanische Medizin (IGTJM e.V.). 